\title{
Self-Assembled Fibrous Nano-Object Generated from Coiled-Coil Polypeptide Diblock Copolymer
}

\author{
Nobuyuki Higashi ${ }^{1}$, Yuichi Gondo ${ }^{1}$, Takahiro Matsumoto ${ }^{1}$ and Tomoyuki Koga ${ }^{1}$ \\ ${ }^{1}$ Department of Molecular Chemistry and Biochemistry, Faculty of Science \& Engineering, \\ Doshisha University, Kyotanabe, Kyoto 610-0321, JAPAN \\ Tel:+81-774-65-6622, Fax: +81-774-65-6844, e-mail: nhigashi@mail.doshisha.ac.jp
}

\begin{abstract}
A zwitterionic diblockpolypeptide of poly(L-lysine)-b-poly(L-glutamic acid) having 20 residues as the segment length of both blocks was synthesized and its self-assembling behavior in water was analyzed in detail. It was evidenced that nanofibers based on a coiled-coil-like motif was produced by accompanying with the sigmoidal transition in the secondary structure of blockpolypeptides. Such transition would generate a staggered helix-dimer with sticky-end and it was rearranged to the one-dimensional nanofiber structure.
\end{abstract}

Key words: Self-assembly, Nanofiber, $\alpha$-helix, Diblock Peptide, Electrostatic Interaction

\section{INTRODUCTION.}

Diblock copolymers comprise two chemically different homopolymers linked together covalently. In most cases, self-assembly occurs when the copolymer is dissolved in a good solvent for only one of the segments due to the chemical incompatibility between the blocks. A variety of morphologies can be formed from the blockpolymers through self-assembly, the most commonly reported being micelles, ${ }^{1,2}$ vesicles, ${ }^{3}$ or syrinders. ${ }^{4}$ In this paper, we report for the first time the formation of nanofiber structures on the basis of a coiled-coil superhelical motif from a polypeptide diblock copolymer. Even if some block copolypeptides have been already synthesized, especially by Deming, ${ }^{5-7}$ the self-assembly of such systems that combine two different polypeptide segments to form a zwitterionic diblock copolymer, poly(L-lysine)- $b$-poly(L-glutamic acid)(PLL- $b$-PLGA), has never been studied, except for the report of Lecommandoux's group. ${ }^{8}$ They have used the same combination of polypeptides and showed the formation of schizophrenic vesicles that can be reversibly produced by alternatively changing $\mathrm{pH}$ between acidic and basic regions. Unfortunately, no morphological exploration has been done in the neutral $\mathrm{pH}$ region, where each segment is ionized negatively and positively, respectively. As an attempt to fabricate peptide nanofiber architectures using self-assembling technique, our group recently focused on the amphiphilic triblock peptides such as $\mathrm{L}_{4} \mathrm{~K}_{8} \mathrm{~L}_{4}$ that were found to self-assemble into well-developed nanofiber structures based on $\beta$-sheet motifs. ${ }^{9-11}$ Such self-assembly of peptides into $\beta$-sheet fibrillar structures has attracted much attention because of not only their association with neurodegeneration diseases but also their potential as novel nano-scale soft-materials. From this point of view, several types of artificial peptides have been developed to produce $\beta$-sheet-based nanofibers. ${ }^{12-15}$ On the other hand, $\alpha$-helix motifs have found a limited use in fabrication of nanofiber architectures. $^{16-18}$ However, the potential of using helical peptide for the construction of nanofibers remains great, considering the fact that fibrils derived from $\alpha$-helical coiled-coil motifs exist widely in native biological systems such as in an extracellular matrix.

A detailed analysis of conformation and morphology of the $\mathrm{PLL}_{20}-b$-PLGA 20 in water at neutral $\mathrm{pH}$ region was performed by using circular dichroism (CD) and fourier transform infrared (FTIR) spectroscopies and atomic force microscopy (AFM) observation. These studies should provide simple and/or essential insight into the mechanism of peptide aggregation, and be useful for the design of novel peptide-based nano-scaffolds.

\section{EXPERIMENTAL}

2.1 Material.

$\operatorname{Poly}(N \varepsilon$-benzyloxycarbonyl-L-lysine $)-b$ - poly $(\gamma-$ benzyl-L-glutamate)(PZLL- $b$-PBLG) diblock copolymer was synthesized by sequential ring-opening polymerization of the corresponding $\alpha$-amino acid

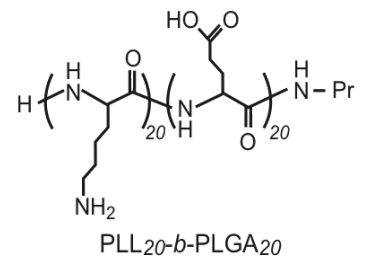

Figure 1. Chemical structure of zwitterionic diblock polypeptide, poly(L-lysine)-block-poly(L-glutamic acid) $\left(\mathrm{PLL}_{20}-b\right.$-PLGA 20 , used as building units for self-assembly. 
$\mathrm{N}$-carboxyanhydrides, according to the modified manner reported previously. ${ }^{19-20}$ The PBLG block was first prepared using $n$-propylamine as the initiator. This led to $\omega$-amino PBLG homopolypeptide from which the PZLL block was grown. Both polymerizations were carried out in DMF at room temperature until the disappearance of acid anhydride bands $\left(1860 \mathrm{~cm}^{-1}, 1790\right.$ $\mathrm{cm}^{-1}$ ) due to monomers in IR spectra. The segment length of both blocks was targeted to be 20 units via monomer/initiator ratio. Finally the protective groups (benzyl and $\mathrm{Z}$ groups) were completely removed after treatment with $25 \% \mathrm{HBr} / \mathrm{CH}_{3} \mathrm{COOH}$ at $50^{\circ} \mathrm{C}$ for $5 \mathrm{~h}$, giving the objective $\mathrm{PLL}_{20}-b$ - $\mathrm{PLGA}_{20}$. For comparison, homopolypeptides ( $\mathrm{PLL}_{20}$ and $\mathrm{PLGA}_{20}$ ) were also prepared by the similar procedure. The self-assembling behavior was examined by means of $\mathrm{CD}$ and FTIR spectroscopies and AFM observation.

\subsection{Measurements.}

CD spectra were recorded on a J-720 spectropolarimeter (JASCO Ltd.) under a nitrogen atmosphere. Experiments were performed in a quartz cell with a $10 \mathrm{~mm}$ path length over the range of 190-250 $\mathrm{nm}$ at room temperature. Sample solutions were prepared by dissolving the sample peptide with $5 \mathrm{mM}$ phosphate buffer (PBS). Final peptide concentration was kept at $5.0 \times 10^{-5}$ amino acid unit $\mathrm{M}$ in aqueous media. ATR-FTIR spectrum was measured on mica plate with the Nexus 470 (Thermo Nicolet Co.) using a Mercury-Cadmium-Tellurium (MCT) detector (resolution, $4 \mathrm{~cm}^{-1}$; number of scan, 1024). The $\mathrm{PLL}_{20}-b$-PLGA 20 was adsorbed onto mica plate after incubation for 6 days at $\mathrm{pH}$ 7.3.

AFM images were collected at ambient temperature on a Nanoscope IIIa (Digital Instrument, Inc.) operated in a tapping-mode and an amplitude-mode using silicon cantilever $(125 \mu \mathrm{m}$, tip radius $c a .10 \mathrm{~nm})$. An aliquot of $\mathrm{PLL}_{20}-b$-PLGA 20 in water was placed on freshly cleaved mica. After adsorption for 3-5 min, the excess solution was removed by absorption onto filter paper and the samples were stored in a covered container to protect them from contamination until they were imaged (within $1 \mathrm{~h}$ ). A $10 \mu \mathrm{m} \times 10 \mu \mathrm{m}$ scanner was used for imaging. The scanning speed was at a line frequency of $1 \mathrm{~Hz}$, and the original images were sampled at a resolution of $512 \times 512$ points.

\section{RESULTS AND DISCUSSION}

3.1 Conformational Property of Zwitterionic Diblock Polypeptide, $\mathbf{P L L}_{20}-b$-PLGA 20 , in Water.

Conformational properties of $\mathrm{PLL}_{20}-b-\mathrm{PLGA}_{20}$ in PBS were first investigated by means of $C D$ spectroscopy. Figure 2A shows the time-dependence of $\mathrm{CD}$ spectra at $\mathrm{pH} 7.3$ ([peptide] $=5.0 \times 10^{-5}$ unit $\mathrm{M}$ ), at which both of segments are charged. The spectra were recorded as prepared and after 6 days-incubation, respectively. Just after dissolving the copolypeptide into PBS, the spectrum indicates a pattern of random coil conformation. Interestingly, however, the 6 days-incubation leads to a drastic change in the spectra;
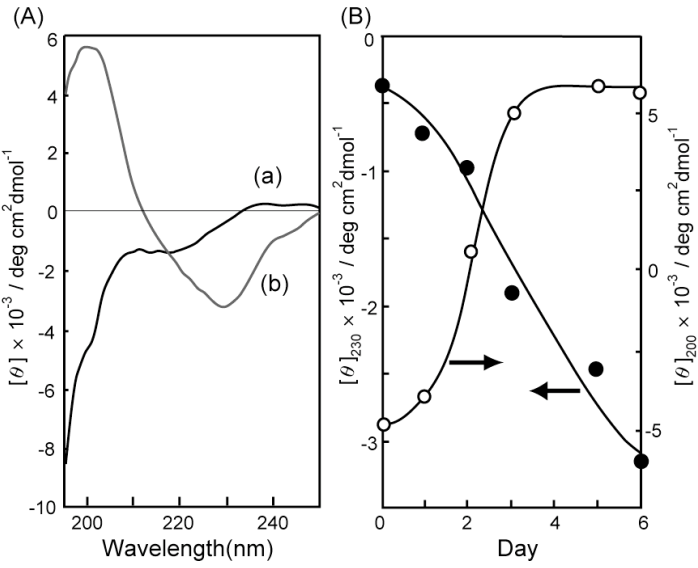

Figure 2. (A) $\mathrm{CD}$ spectra of $\mathrm{PLL}_{20}-b-\mathrm{PLGA}_{20}$ obtained just after sample preparation (a) and after incubation for 6 days (b) in PBS at $\mathrm{pH} 7.3$. [peptide] $=5.0 \times 10^{-5}$ unit M. (B) Plots of molar ellipticity at $230 \mathrm{~nm}\left([\theta]_{230}\right)$ and $200 \mathrm{~nm}\left([\theta]_{200}\right)$ as a function of time.

the negative peak at $200 \mathrm{~nm}$ assigned to the random coil conformation turns to the positive one, and simultaneously the negative peak at around $230 \mathrm{~nm}$ and the shoulder at around $210 \mathrm{~nm}$ newly appear. The spectrum after incubation is not a typical pattern of isolated $\alpha$-helix conformation that normally provides negative peaks at $222 \mathrm{~nm}$ and $208 \mathrm{~nm}$, but the observed red-shifting and flattening should be assigned to the formation of $\alpha$-helix aggregation. ${ }^{21}$ Figure 2B shows the plot of molar ellipticity at $230 \mathrm{~nm}\left([\theta]_{230}\right)$ and 200 $\mathrm{nm}\left([\theta]_{200}\right)$ as a function of time. With incubation, the $[\theta]_{230}$ value decreased. In contrast, $[\theta]_{200}$ value sigmoidally increased with incubation, and reached constant values after 6 days. ATR-FTIR spectrum of

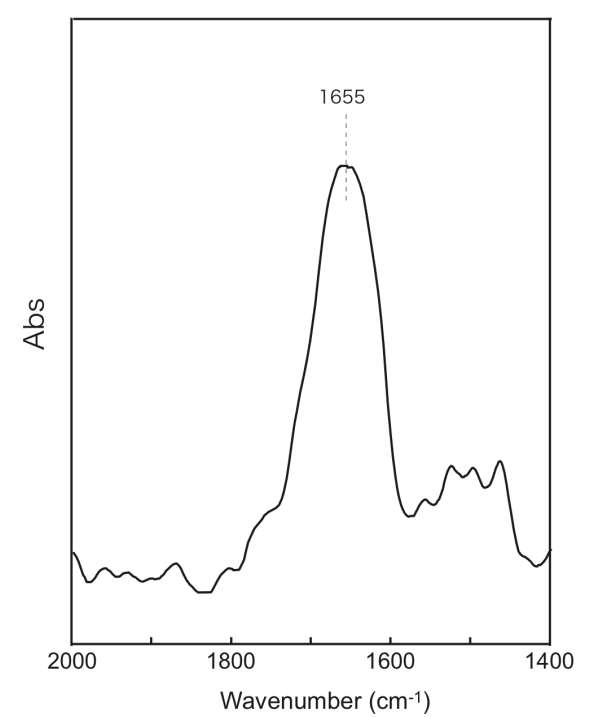

Figure 3. ATR-FTIR spectrum of $\mathrm{PLL}_{20}-b-\mathrm{PLGA}_{20}$ on mica substrate obtained after incubation for 6 days in PBS at $\mathrm{pH}$ 7.3. [peptide] $=5.0 \times 10^{-5}$ unit $\mathrm{M}$. 
$\mathrm{PLL}_{20}-b$-PLGA 20 obtained after incubation for 6 days supports the $\mathrm{CD}$ results and provides the information on the secondary structure. In the amide I region, characteristic absorption of an $\alpha$-helix structure ${ }^{22}$ was observed at $1655 \mathrm{~cm}^{-1}$ (Figure 3 ). When the same experiments were performed at acidic and basic $\mathrm{pHs}$ (pHs 3.2 and 11.3), at which both peptide segments of $\mathrm{PLL}_{20}-b-\mathrm{PLGA}_{20}$ are protonated and deprotonated, respectively, CD spectra gave a random coil pattern (including small amount of $\alpha$-helix structure) and did not show significant change even after 6 days at both of pHs. AFM images of these samples obtained after 6 days-incubation (data not shown) showed spherical aggregates with diameters of around 100-200 nm, suggesting the formation of vesicles as was previously described by Lecommandoux's group. ${ }^{8}$

\subsection{Self-assembling Property of Zwitterionic Diblock Polypeptide, PLL $_{20}-b$-PLGA $A_{20}$, in Water at Neutral pH.}

The time-dependence of AFM images prepared from $\mathrm{PLL}_{20}-b-\mathrm{PLGA}_{20}$ at $\mathrm{pH} 7.3$ is displayed in Figure 4. Globular aggregates are observed immediately after preparation of the sample solution (Figure 4a), resulting from a rapid, heterogeneous aggregation through electrostatic interactions among the oppositely-charged segments. Similar AFM image of globular aggregates was obtained after 2 days-incubation. After incubation for 6 days, it can be clearly seen in the image that fibrous object has newly grown from the globular aggregates (Figure 4b). This nanofiber has a left-handed twist that repeats along the fiber axis (Figure $4 \mathrm{c}$, amplitude image). The periodicity of this repeat is about $30 \mathrm{~nm}$ and the fiber height is estimated to be $1.0 \pm$ $0.3 \mathrm{~nm}$. It should be noted that time-course of the
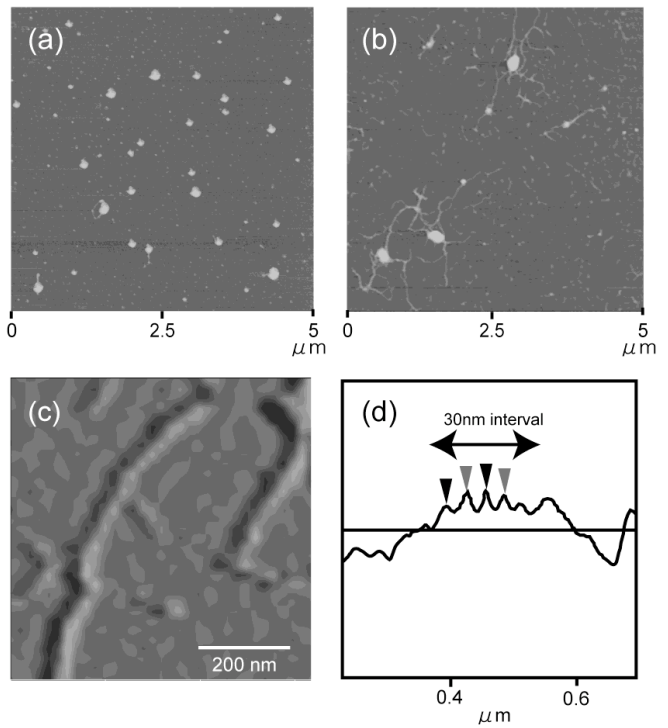

Figure 4. AFM images of $\mathrm{PLL}_{20}-b-\mathrm{PLGA}_{20}$ obtained just after sample preparation (a) and after 6 days-incubation (b) in PBS at pH 7.3. Amplitude image (c) and its analysis (d) corresponding to the nanofiber. [peptide] $=5.0 \times 10^{-5}$ unit M.

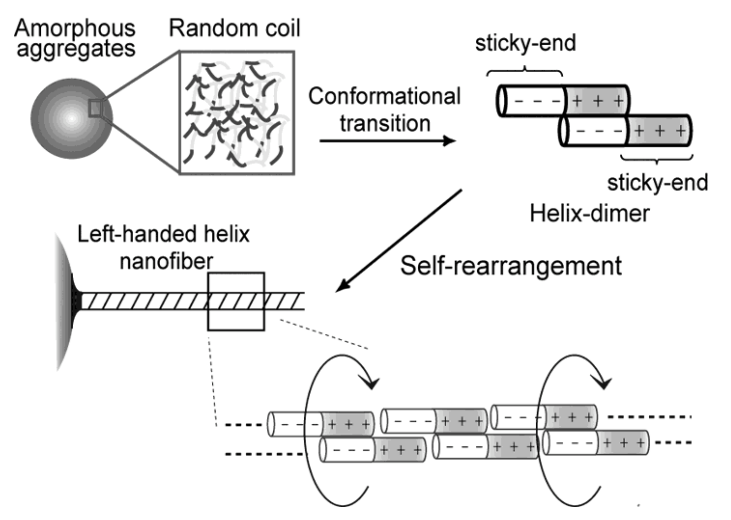

Figure 5. Schematic illustration for the formation mechanism of $\mathrm{PLL}_{20}-b$-PLGA 20 -nanofiber at neutral $\mathrm{pH}$ region.

nanofiber formation does not agree well with that of conformational transition. Considering both of these AFM results and the above CD spectroscopic data, a coiled-coil structure as shown schematically in Figure 5 may be most favorable to explain the process of nanofiber formation. A coiled-coil is well-known to be a left-handed superhelical structure consisting of a bundle of ionic, right-handed $\alpha$-helices wrapped around each other. At $\mathrm{pH}$ 7.3, ionized PLL and PLGA segments of $\mathrm{PLL}_{20}-b$ - $\mathrm{PLGA}_{20}$ rapidly form amorphous, globular and huge aggregates through electrostatic interaction between them. However, since both of PLL and PLGA segments themselves are originally inherent in $\alpha$-helical conformation in aqueous media, the partial neutralization of ionized segments due to inter-polypeptide complexation slowly induces the conformational transition from random coil to $\alpha$-helix. This conformational transition would generate a staggered helix-dimer with "sticky-end" based on its block structure and the dimers are rearranged to the one-dimensional nanofiber structure by tagging their sticky-ends, which must be energetically more stable state (Figure 5). In fact, such nanofiber formation was not observed in the system of an equimolar mixture of $\mathrm{PLL}_{20}$ and $\mathrm{PLGA}_{20}$ homopolypeptides, but amorphous aggregates with $\beta$-sheet conformation were produced rapidly after sample preparation, probably due to their "blunt-end" type complexation. To elucidate the importance of ionic interactions contributing to trigger and stabilize the coiled-coil structure, $\mathrm{CD}$ and AFM measurements of $\mathrm{PLL}_{20}-b$ - $\mathrm{PLGA}_{20}$ were undertaken in the presence of $200 \mathrm{mM} \mathrm{NaCl}$ and the other conditions were the same as those mentioned above. As a result, the $[\theta]_{230}$ value showed only slight decrease even after 6 days and there observed no fibril structure in AFM image.

\section{CONCLUSION}

In summary, new zwitterionic blockpolypeptide was synthesized, and its original self-assembling behavior in water of neutral $\mathrm{pH}$ was examined in detail. We evidenced here for the first time the formation of 
coiled-coil-like nanofibers that can be produced by accompanying with the sigmoidal transition in the secondary structure from random coil to $\alpha$-helix of blockpolypeptides. The coiled-coil-like $\alpha$-helix-based nanofiber formation from simple copolypeptides will open an avenue to the understanding of a complex higher order structure of protein and to the construction of novel peptide-based nanomaterials.

\section{ACKNOWLEDGEMENT}

This work was supported in part by a grant-in-aid for young scientific research (No. 19710099, 21750130) from the Ministry of Education, Culture, Sports, Science and Technology (MEXT) Japan, and by the Projects on "Creating Research Center for Advanced Molecular Biochemistry" and "Research Center for Interfacial Phenomena" from the MEXT and Doshisha Univ.

\section{REFERENCES}

[1] A. Guo, G. Liu, J. Tao, Macromolecules 1996, 29, 2487.

[2] G. Riess, Prog. Polym. Sci. 2003, 28, 1107.

[3] S. Burke, H. Shen, A. Eisenberg, Macromol. Symp. 2001, 175, 273.

[4] S. Stewart, G. Liu, Angew. Chem. Int. Ed. 2000, 39, 340.

[5] T. J. Deming, Macromolecules 1999, 32, 4500.

[6] A. P. Nowak, V. Breedveld, L. Pakstis, B. Ozbas, D. J. Pine, D. Pochan, T. J. Deming, Nature 2002, 417, 424.

[7] E. Bellomo, M. D. Wyrsta, L. Pakstis, D. J. Pochan,

T. J. Deming, Nat. Mater. 2004, 3, 244.

[8] J. Rodríguez-Hernández, S. Lecommandoux, J. Am. Chem. Soc. 2005, 127, 2026.

[9] T. Koga, M. Higuchi, T. Kinoshita, N. Higashi, Chem. Eur. J. 2006, 12, 1360.

[10] T. Koga, M. Matsuoka, N. Higashi, J. Am. Chem. Soc. 2005, 127, 17596.

[11] T. Koga, K. Kitamura, N. Higashi, Chem. Commun. 2006, 4896.

[12] J. D. Hartgerink, E. Beniash, S. I. Stupp, Science 2001, 294, 1684.

[13] H. A. Lashuel, S. R. LaBrenz, L. Woo, L. C. Serpell, L. W. Kelly, J. Am. Chem. Soc. 2000, 122, 5262.

[14] Y. Takahashi, A. Ueno, H. Mihara, Chem. Eur. J. 1998, 4, 2475.

[15] H. Yokoi, T. Kinoshita, and S. Zhang, Proc. Natl. Acad. Sci. USA 2005, 102, 8414.

[16] M. J. Pandya, G. M. Spooner, M. Sunde, J. R. Thorpe, A. Rodger, D. N. Woolfson, Biochemistry 2000, 39,8728 .

[17] M. G. Ryadnov and D. N. Woolfson, Angew. Chem. Int. Ed. 2003, 42, 3021.

[18] M. G. Ryadnov and D. N. Woolfson, Nat. Mater. 2003, 2, 329.

[19] N. Higashi, T. Koga, M. Niwa, Langmuir 2000, 16, 3482.

[20] N. Higashi, A. Uchino, Y. Mizuguchi, M. Niwa, J. Biol. Macromol. 2006, 38, 120.
[21] H. Maeda, H. Kato, S. Ikeda, Biopoplymers 1976, 23, 1333 .

[22] T. Miyazawa, E. R. Blout, J. Am. Chem. Soc. 1961, 83,712 .

(Received January 29, 2010; Accepted March 14, 2010) 